\title{
Stratifications of teardrops
}

\author{
by
}

Bruce Hughes (Nashville, TN)

\begin{abstract}
Teardrops are generalizations of open mapping cylinders. We prove that the teardrop of a stratified approximate fibration $X \rightarrow Y \times \mathbb{R}$ with $X$ and $Y$ homotopically stratified spaces is itself a homotopically stratified space (under mild hypothesis). This is applied to manifold stratified approximate fibrations between manifold stratified spaces in order to establish the realization part of a previously announced tubular neighborhood theory.
\end{abstract}

1. Introduction. The teardrop of a map $p: X \rightarrow Y \times \mathbb{R}$ is a space $X \cup_{p} Y$ obtained by gluing $Y$ to the positive infinity end of $X$ determined by the map $p$. Such a construction is necessary to describe neighborhoods of $Y$ in a space $Z$ when $Y$ may fail to have a mapping cylinder neighborhood.

We work in the general setting of the homotopically stratified spaces of Quinn [15]. The failure to have mapping cylinder neighborhoods is fairly common for such spaces, even when the strata are manifolds. In [6] a theory of tubular neighborhoods for these manifold stratified spaces was announced (for high-dimensional strata). The existence part of the theory says that every skeleton $Y$ has a neighborhood which is the teardrop of some manifold stratified approximate fibration $X \rightarrow Y \times \mathbb{R}$. The uniqueness part says that the manifold stratified approximate fibration is unique up to controlled, stratum preserving homeomorphism.

The goal of this paper is to give the realization part of the theory. Namely, we prove that if $Y$ is a potential skeleton of a manifold stratified space and $p: X \rightarrow Y \times \mathbb{R}$ is a manifold stratified approximate fibration, then $X \cup_{p} Y$ is a manifold stratified space with $Y$ as a skeleton. This is the third part of

1991 Mathematics Subject Classification: Primary 57N80, 55R65; Secondary 57N40, $58 \mathrm{~A} 35$.

Key words and phrases: stratified space, teardrop, homotopically stratified space, stratified approximate fibration, mapping cylinder, manifold stratified space.

Supported in part by NSF Grant DMS-9504759. 
the Main Theorem. The first two parts show that weaker conditions on $X$ and $Y$ are also reflected in the teardrop.

MAIN TheOREM. Let $p: X \rightarrow Y \times \mathbb{R}$ be a proper stratified approximate fibration between locally compact, separable metric spaces with stratifications. Suppose that $X$ and $Y$ have only finitely many strata and that the strata of $Y$ are path connected.

(1) If $X$ and $Y$ are homotopically stratified, then so is $X \cup_{p} Y$.

(2) If , in addition, $X$ and $Y$ have ANR strata and compactly dominated local holinks, then so does $X \cup_{p} Y$.

(3) If , in addition, $X$ and $Y$ are manifold stratified, then so is $X \cup_{p} Y$.

The three parts of this theorem appear in Theorems 4.3, 5.4 and 6.1 below.

The work [11] by Hughes, Taylor, Weinberger, and Williams contains a complete account of the tubular neighborhood theory for manifold stratified spaces with two strata. The papers [7] and [8], as well as the present paper, are installments in the series (to culminate in [9]) which will provide the theory in the general multiple strata case.

Even though certain aspects of [11] and [8] are generalized here, it is important to understand that the present paper depends on many of the results in the previous two (as well as [7]).

2. Definitions. In this section we gather some background material on mapping cylinders, teardrops, spaces with stratifications, and stratified approximate fibrations.

Mapping cylinders. The mapping cylinder of a map $p: X \rightarrow Y$ is the space

$$
\operatorname{cyl}(p)=((X \times I) \amalg Y) /\{(x, 1) \sim p(x) \in Y \mid x \in X\}
$$

with the teardrop topology, that is, the minimal topology such that:

(1) the inclusion $X \times[0,1) \rightarrow \operatorname{cyl}(p)$ is an open embedding,

(2) the function

$$
c: \operatorname{cyl}(p) \rightarrow Y \times I, \quad \begin{cases}{[x, t] \mapsto(p(x), t)} & \text { if }(x, t) \in X \times[0,1), \\ {[y] \mapsto(y, 1)} & \text { if } y \in Y,\end{cases}
$$

is continuous.

The open mapping cylinder $\operatorname{cyl}(p)$ is $\operatorname{cyl}(p) \backslash(X \times\{0\})$. If $p: X \rightarrow Y$ is a proper map between locally compact Hausdorff spaces, then the teardrop topology agrees with the usual quotient space topology on the mapping cylinder. See $[10$, Chap. 12$]$ and $[11, \S 3]$ for further remarks on the teardrop topology. 
We will have occasion to use the following three maps:

$$
\begin{aligned}
& p_{X}: \operatorname{cyl}(p) \backslash Y \rightarrow X, \quad[x, t] \mapsto x, \quad(x, t) \in X \times[0,1), \\
& p_{Y}: \operatorname{cyl}(p) \rightarrow Y, \quad \begin{cases}{[x, t] \mapsto p(x),} & (x, t) \in X \times I, \\
{[y] \mapsto y,} & y \in Y,\end{cases} \\
& p_{I}: \operatorname{cyl}(p) \rightarrow I, \quad \begin{cases}{[x, t] \mapsto t,} & (x, t) \in X \times I, \\
{[y] \mapsto 1,} & y \in Y .\end{cases}
\end{aligned}
$$

Teardrops. The teardrop of a map $p: X \rightarrow Y \times \mathbb{R}$ is the space denoted by $X \cup_{p} Y$ whose underlying set is the disjoint union $X \amalg Y$ with the minimal topology such that

(1) $X \subseteq X \cup_{p} Y$ is an open embedding, and

(2) the function $c: X \cup_{p} Y \rightarrow Y \times(-\infty,+\infty]$, called the teardrop collapse, defined by

$$
c(x)= \begin{cases}p(x) & \text { if } x \in X, \\ (x,+\infty) & \text { if } x \in Y\end{cases}
$$

is continuous.

This generalizes the open mapping cylinder of a map $g: X \rightarrow Y$. Namely, cyl $(g)$ is naturally homeomorphic to the teardrop $(X \times \mathbb{R}) \cup_{g \times i d_{\mathbb{R}}} Y$. However, not all teardrops are open mapping cylinders because not all maps to $Y \times \mathbb{R}$ can be split as a product.

Spaces with stratifications. The basic definitions from the theory of stratifications are presented here. For a fuller treatment see [7].

(1) A partition of a space $X$ consists of an index set $\mathcal{I}$ and a collection $\left\{X_{i}\right\}_{i \in \mathcal{I}}$ of pairwise disjoint subspaces of $X$ such that $X=\bigcup_{i \in \mathcal{I}} X_{i}$.

(2) A stratification of a space $X$ consists of an index set $\mathcal{I}$ and a locally finite partition $\left\{X_{i}\right\}_{i \in \mathcal{I}}$ of locally closed subspaces of $X$.

(3) In either case, for $i \in \mathcal{I}, X_{i}$ is the $i$-stratum and

$$
X^{i}=\bigcup\left\{X_{k} \mid X_{k} \cap \operatorname{cl}\left(X_{i}\right) \neq \emptyset\right\}
$$

is the $i$-skeleton.

For a space $X$ with a stratification $\left\{X_{i}\right\}_{i \in \mathcal{I}}$, define a relation $\leq$ on the index set $\mathcal{I}$ by

$$
i \leq j \quad \text { if and only if } \quad X_{i} \subseteq \operatorname{cl}\left(X_{j}\right) .
$$

The stratification satisfies the Frontier Condition if for every $i, j \in \mathcal{I}$,

$$
X_{i} \cap \operatorname{cl}\left(X_{j}\right) \neq \emptyset \quad \text { implies } \quad X_{i} \subseteq \operatorname{cl}\left(X_{j}\right) .
$$

If $\left\{X_{i}\right\}_{i \in \mathcal{I}}$ is a stratification of $X$, then the Frontier Condition holds if and only if $\leq$ is a partial ordering of $\mathcal{I}$ and for each $i \in \mathcal{I}, X^{i}=\operatorname{cl}\left(X_{i}\right)$. 
Let $X$ be a space with a partition.

(1) A map $f: Z \times A \rightarrow X$ is stratum preserving along $A$ if for each $z \in Z, f(\{z\} \times A)$ lies in a single stratum of $X$.

(2) A map $f: Z \times I \rightarrow X$ is a stratum preserving homotopy if $f$ is stratum preserving along $I$.

(3) A map $f: Z \times I \rightarrow X$ is a nearly stratum preserving homotopy if $f \mid Z \times[0,1)$ is stratum preserving along $[0,1)$.

(4) If $A, Y \subseteq X$, then a map $f: Y \times I \rightarrow X$ is a nearly stratum preserving deformation of $Y$ to $A$ in $X$ if $f_{0}=$ inclusion, $f$ is a nearly stratum preserving homotopy, and $f_{1}(Y) \subseteq A$.

The natural partitions of mapping cylinders and teardrops. Let $X$ and $Y$ be spaces with partitions $\left\{X_{i}\right\}_{i \in \mathcal{I}}$ and $\left\{Y_{j}\right\}_{j \in \mathcal{J}}$, respectively. If $p: X \rightarrow Y$ is a map, then the mapping cylinder $\operatorname{cyl}(p)$ is naturally partitioned by

$$
\left\{X_{i} \times[0,1)\right\}_{i \in \mathcal{I}} \cup\left\{Y_{j}\right\}_{j \in \mathcal{J}} .
$$

If $p: X \rightarrow Y \times \mathbb{R}$ is a map, then the teardrop $X \cup_{p} Y$ is naturally partitioned by

$$
\left\{X_{i}\right\}_{i \in \mathcal{I}} \cup\left\{Y_{j}\right\}_{j \in \mathcal{J}}
$$

The product $Y \times \mathbb{R}$ is naturally partitioned by $\left\{Y_{j} \times \mathbb{R}\right\}_{j \in \mathcal{J}}$. Moreover, $Y \times(-\infty,+\infty]$ is naturally partitioned by $\left\{Y_{j} \times \mathbb{R}\right\}_{j \in \mathcal{J}} \cup\left\{Y_{j} \times\{+\infty\}\right\}_{j \in \mathcal{J}}$. Thus, $Y \times(-\infty,+\infty]$ is the open mapping cylinder of the identity $Y \rightarrow Y$ with the natural partition.

Stratified approximate fibrations. More definitions are recalled from [6] and [7]. Let $X$ and $Y$ be spaces with partitions $\left\{X_{i}\right\}_{i \in \mathcal{I}}$ and $\left\{Y_{j}\right\}_{j \in \mathcal{J}}$, respectively. A map $p: X \rightarrow Y$ is a stratified approximate fibration provided given any commuting diagram

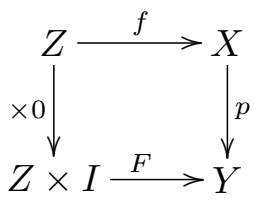

with $F$ a stratum preserving homotopy, there exists a stratified controlled solution, i.e., a map $\widetilde{F}: Z \times I \times[0,1) \rightarrow X$ which is stratum preserving along $I \times[0,1)$ and such that $\widetilde{F}(z, 0, t)=f(z)$ for each $(z, t) \in Z \times[0,1)$, and the function $\bar{F}: Z \times I \times I \rightarrow Y$ defined by $\bar{F} \mid Z \times I \times[0,1)=p \widetilde{F}$ and $\bar{F} \mid Z \times I \times\{1\}=F \times \operatorname{id}_{\{1\}}$ is continuous and stratum preserving along $I \times I$.

Of course, an approximate fibration is a map $p: X \rightarrow Y$ which is a stratified approximate fibration when $X$ and $Y$ are stratified with a single stratum. For more background on approximate fibrations from the point of 
view used here see [12]. The original definition of an approximate fibration is due to Coram and Duvall [3].

3. Embedding a teardrop in a mapping cylinder. In this section we show how to embed teardrops into certain mapping cylinders. This allows us to use our previous results on mapping cylinders [8] to prove results about teardrops in $\S 4$. We also prove a technical result about mapping cylinders which will be used in $\S 4$.

Let $X$ and $Y$ be spaces with partitions $\left\{X_{i}\right\}_{i \in \mathcal{I}}$ and $\left\{Y_{j}\right\}_{j \in \mathcal{J}}$, respectively. Let $p: X \rightarrow Y \times \mathbb{R}$ be a proper map. Consider the composition

$$
p^{\prime}: X \stackrel{p}{\longrightarrow} Y \times \mathbb{R} \stackrel{\subseteq}{\longrightarrow} Y \times(-\infty,+\infty] .
$$

Let $\beta: Y \times(-\infty,+\infty] \rightarrow[0,1]$ be a map such that $\beta^{-1}(1)=Y \times\{+\infty\}$. Consider the subspace $\operatorname{cyl}\left(p^{\prime}\right)_{\beta} \subseteq \operatorname{cyl}\left(p^{\prime}\right)$ defined by

$$
\operatorname{cyl}\left(p^{\prime}\right)_{\beta}=\left\{[x, s] \in \operatorname{cyl}\left(p^{\prime}\right) \mid \beta p^{\prime}(x) \leq s \leq 1\right\} \cup Y \times(-\infty,+\infty] .
$$

This is a damped mapping cylinder. Define $\alpha_{\beta}: X \cup_{p} Y \rightarrow \operatorname{cyl}\left(p^{\prime}\right)_{\beta}$ by

$$
\begin{cases}\alpha_{\beta}(x)=[x, \beta(p(x))] \in \operatorname{cyl}\left(p^{\prime}\right) & \text { if } x \in X \\ \alpha_{\beta}(y)=[y,+\infty] \in Y \times\{+\infty\} \subseteq \operatorname{cyl}\left(p^{\prime}\right) & \text { if } y \in Y .\end{cases}
$$

Proposition 3.1. $\alpha_{\beta}: X \cup_{p} Y \rightarrow \operatorname{cyl}\left(p^{\prime}\right)_{\beta}$ is a stratum preserving embedding.

Proof. To see that $\alpha_{\beta}$ is continuous, apply the Continuity Criteria $[11,3.4]$. To this end, first observe that

$$
\alpha_{\beta} \mid \alpha_{\beta}^{-1}\left(\operatorname{cyl}\left(p^{\prime}\right)_{\beta} \backslash Y \times(-\infty,+\infty]\right) \subseteq X \rightarrow \operatorname{cyl}\left(p^{\prime}\right)_{\beta} \backslash Y \times(-\infty,+\infty]
$$

is given by $\alpha_{\beta}(x)=(x, \beta p(x))$, hence, is continuous. Then observe that the composition $c \alpha_{\beta}: X \cup_{p} Y \rightarrow Y \times(-\infty,+\infty] \times I$, where $c: \operatorname{cyl}\left(p^{\prime}\right)_{\beta} \rightarrow$ $Y \times(-\infty,+\infty] \times I$ is the restriction of the teardrop collapse for $\operatorname{cyl}\left(p^{\prime}\right)$, is just the map $c^{\prime} \times \beta c^{\prime}$, where $c^{\prime}: X \cup_{p} Y \rightarrow Y \times(-\infty,+\infty]$ is the teardrop collapse for $X \cup_{p} Y$; hence, $\alpha_{\beta}$ is continuous.

Obviously, $\alpha_{\beta}$ is one-to-one. To see that $\alpha_{\beta}^{-1}$ is continuous, note that $\operatorname{Im}\left(\alpha_{\beta}\right) \subseteq X \times[0,1) \cup Y \times\{+\infty\} \subseteq \operatorname{cyl}\left(p^{\prime}\right)$. Define $\gamma: X \times[0,1) \cup Y \times\{+\infty\} \rightarrow$ $X \cup_{p} Y$ by

$$
\gamma(x, s)=x \in X \subseteq X \cup_{p} Y, \quad \gamma(y,+\infty)=y \in Y \subseteq X \cup_{p} Y .
$$

Then $\gamma$ is continuous (use $[11,3.4]$ again) and $\alpha_{\beta}^{-1}=\gamma \mid \operatorname{Im}\left(\alpha_{\beta}\right)$.

That $\alpha_{\beta}$ preserves strata is immediate.

The following result will be used in the proof of Lemma 4.5.

Proposition 3.2. If $X$ and $Y$ are locally compact, separable, metric spaces with stratifications satisfying the Frontier Condition and $p: X \rightarrow$ 
$Y \times \mathbb{R}$ is a proper stratified approximate fibration, then there exists a nearly stratum preserving deformation of $\operatorname{cyl}\left(p^{\prime}\right)_{\beta}$ to $Y \times\{+\infty\}$ rel $Y \times\{+\infty\}$.

Proof. The conditions on $Y$ imply that it can be given a complete bounded metric $d$. Assume that $Y \times(-\infty,+\infty]$ has a product metric so that projection $Y \times(-\infty,+\infty] \rightarrow Y$ is distance nonincreasing. Recall from $[11,3.15]$ that $\operatorname{cyl}\left(p^{\prime}\right)_{\beta}$ is metrizable. We begin by giving a convenient description of a nearly stratum preserving deformation $g: Y \times(-\infty,+\infty] \times I \rightarrow$ $Y \times(-\infty,+\infty]$ of $Y \times(-\infty,+\infty]$ to $Y \times\{+\infty\}$ rel $Y \times\{+\infty\}$. For each $i=0,1, \ldots$, let

$$
g^{i}: Y \times(-\infty,+\infty] \times\left[\frac{i}{i+1}, \frac{i+1}{i+2}\right] \rightarrow Y \times(-\infty,+\infty]
$$

be an isotopy such that

(1) $g_{i /(i+1)}^{i}=\mathrm{id}$,

(2) $g^{i}$ is supported on $Y \times(-\infty, i+0.75]$,

(3) $\operatorname{Im}\left(g_{(i+1) /(i+2)}^{i}\right) \subseteq Y \times[i+0.25,+\infty]$,

(4) $g^{i}$ is fiber preserving over $Y$.

Then define $g: Y \times(-\infty,+\infty] \times I \rightarrow Y \times(-\infty,+\infty]$ inductively by

$$
g_{t}=g_{t}^{i} \circ g_{i /(i+1)}^{i-1} \circ \ldots \circ g_{1 / 2}^{0} \quad \text { for } \frac{i}{i+1} \leq t \leq \frac{i+1}{i+2},
$$

and $g_{1}(y, s)=(y,+\infty)$.

Using the fact that $p$ is a proper stratified approximate fibration and the interpretation of controlled maps in [11,3.19], one can deduce the existence of maps

$$
\widetilde{g}^{i}: \operatorname{cyl}(p) \times\left[\frac{i}{i+1}, \frac{i+1}{i+2}\right] \rightarrow \operatorname{cyl}(p),
$$

for each $i=0,1, \ldots$, such that

(1) $\widetilde{g}_{i /(i+1)}^{i}=\mathrm{id}$

(2) $\widetilde{g}^{i}$ is stratum preserving along $[i /(i+1),(i+1) /(i+2)]$,

(3) $\widetilde{g}^{i}$ is level preserving, i.e., $p_{I} \widetilde{g}^{i}=p_{I}$,

(4) $p_{Y \times \mathbb{R}} \circ \widetilde{g}_{t}^{i}$ is $1 / 2^{i}$-close to $g_{t}^{i} \circ p_{Y \times \mathbb{R}}$ for each $t \in[i /(i+1)$, $(i+1) /(i+2)]$,

(5) $p_{Y \times \mathbb{R}} \circ \widetilde{g}_{t}^{i} \mid p_{Y \times \mathbb{R}}^{-1}(Y \times[n,+\infty))$ is $1 / 2^{n}$-close to $g_{t}^{i} \circ p_{Y \times \mathbb{R}} \mid$ for each $t \in[i /(i+1),(i+1) /(i+2)]$ and $n=0,1, \ldots$,

(6) $\widetilde{g}^{i}\left|Y \times \mathbb{R} \times I=g^{i}\right| Y \times \mathbb{R} \times I$.

Extend $\widetilde{g}^{i}$ to a map with the same name

$$
\widetilde{g}^{i}: \operatorname{cyl}\left(p^{\prime}\right) \times\left[\frac{i}{i+1}, \frac{i+1}{i+2}\right] \rightarrow \operatorname{cyl}\left(p^{\prime}\right)
$$


via the identity on $Y \times\{+\infty\}$. In order to show that this extension is continuous, suppose $\left\{\left(w_{j}, t_{j}\right)\right\}$ is a sequence in $\operatorname{cyl}(p) \times[i /(i+1),(i+1) /(i+2)]$ such that $w_{j} \rightarrow\left(y_{0},+\infty\right) \in Y \times\{+\infty\}$ and $t_{j} \rightarrow t_{0}$ as $j \rightarrow \infty$. It must be shown that

$$
\widetilde{g}^{i}\left(w_{j}, t_{j}\right) \rightarrow \widetilde{g}^{i}\left(y_{0},+\infty, t_{0}\right)=\left(y_{0},+\infty\right) \quad \text { as } j \rightarrow \infty .
$$

Because of the teardrop topology and the fact that $\widetilde{g}^{i}$ is level preserving, it suffices to show that $p_{Y \times \mathbb{R}} \circ \widetilde{g}_{t_{j}}^{i}\left(w_{j}\right) \rightarrow\left(y_{0},+\infty\right)$ as $j \rightarrow \infty$. But item (5) implies that the distance between $p_{Y \times \mathbb{R}} \circ \widetilde{g}_{t_{j}}^{i}\left(w_{j}\right)$ and $g_{t_{j}}^{i} \circ p_{Y \times \mathbb{R}}\left(w_{j}\right)$ converges to 0 as $j \rightarrow \infty$. Also, $p_{Y \times \mathbb{R}}\left(w_{j}\right) \rightarrow\left(y_{0},+\infty\right)$ as $j \rightarrow \infty$. Thus,

$$
g^{i}\left(p_{Y \times \mathbb{R}}\left(w_{j}\right), t_{j}\right) \rightarrow g^{i}\left(y_{0},+\infty, t_{0}\right)=\left(y_{0},+\infty\right) \quad \text { as } j \rightarrow \infty .
$$

This completes the proof that $\widetilde{g}^{i}$ is continuous.

Define $\widetilde{g}: \operatorname{cyl}\left(p^{\prime}\right) \times[0,1) \rightarrow \operatorname{cyl}\left(p^{\prime}\right)$ inductively by

$$
\widetilde{g}_{t}=\widetilde{g}_{t}^{i} \circ \widetilde{g}_{i /(i+1)}^{i-1} \circ \cdots \circ \widetilde{g}_{1 / 2}^{0} \quad \text { for } \frac{i}{i+1} \leq t \leq \frac{i+1}{i+2} .
$$

In particular, $\widetilde{g}$ is stratum preserving along $[0,1)$.

Let $\varrho: \operatorname{cyl}\left(p^{\prime}\right) \rightarrow \operatorname{cyl}\left(p^{\prime}\right)_{\beta}$ be the natural retraction defined by pushing down the mapping cylinder rays. Let $p_{\infty}: \operatorname{cyl}\left(p^{\prime}\right) \rightarrow Y \times\{+\infty\}$ be given by

$$
p_{\infty}=\text { projection } \circ p_{Y \times(-\infty,+\infty]} \text {. }
$$

Define

$$
\widehat{g}: \operatorname{cyl}\left(p^{\prime}\right)_{\beta} \times I \rightarrow \operatorname{cyl}\left(p^{\prime}\right)_{\beta}, \quad\left\{\begin{array}{l}
\widehat{g} \mid \operatorname{cyl}\left(p^{\prime}\right)_{\beta} \times[0,1)=\varrho \circ \widetilde{g}, \\
\widehat{g}_{1}=\lim _{t \rightarrow 1} p_{\infty} \circ \widetilde{g}_{t} .
\end{array}\right.
$$

Note that $g \mid Y \times(-\infty,+\infty] \times I=g$.

We need to conclude that $\widehat{g}_{1}$ is well defined and that $\widehat{g}$ is continuous. Using the fact that the $g^{i}$ are fiber preserving over $Y$ and the definition of $\widetilde{g}$, one can verify that: for every $\varepsilon>0$ there exists $t_{0}<1$ such that $d\left(p_{\infty} \widetilde{g}_{t}, p_{\infty} \widetilde{g}_{s}\right)<\varepsilon$ if $t_{0} \leq s, t<1$. The desired conclusions then follow from standard facts about complete metric spaces.

4. Homotopically stratified teardrops. In this section we recall the definitions relating to Quinn's homotopically stratified spaces [15], and then prove the main result, Theorem 4.3, showing that the teardrop construction keeps one inside the category of homotopically stratified spaces.

Homotopy links. A homotopy model for the normal space of a subspace is provided by the homotopy link (cf. [15]). Let $X$ be a space with a partition $\left\{X_{i}\right\}_{i \in \mathcal{I}}$ and $Y \subseteq X$.

(1) The homotopy link of $Y$ in $X$ is defined by

$$
\operatorname{holink}(X, Y)=\left\{\omega \in X^{I} \mid \omega(t) \in Y \text { if and only if } t=0\right\} \text {. }
$$


(2) The stratified homotopy link of $Y$ in $X$ consists of all $\omega$ in holink $(X, Y)$ such that $\omega((0,1])$ lies in a single stratum of $X$ :

$\operatorname{holink}_{\mathrm{s}}(X, Y)=\left\{\omega \in \operatorname{holink}(X, Y) \mid\right.$ for some $i, \omega(t) \subseteq X_{i}$ for all $\left.t \in(0,1]\right\}$.

(3) Evaluation at 0 defines maps

$q: \operatorname{holink}(X, Y) \rightarrow Y \quad$ and $\quad q: \operatorname{holink}_{\mathrm{s}}(X, Y) \rightarrow Y, \quad q(\omega)=\omega(0)$,

both called holink evaluation.

(4) The stratified homotopy link has a natural partition with $i$-stratum

$$
\operatorname{holink}_{\mathrm{s}}(X, Y)_{i}=\left\{\omega \in \operatorname{holink}_{\mathrm{s}}(X, Y) \mid \omega(1) \in X_{i}\right\} .
$$

Homotopically stratified spaces. More definitions are now recalled (see [6], [7], [11], [15]). A subset $Y \subseteq X$ is forward tame in $X$ if there exist a neighborhood $U$ of $Y$ in $X$ and a homotopy $h: U \times I \rightarrow X$ such that $h_{0}=$ inclusion $: U \rightarrow X, h_{t} \mid Y=$ inclusion $: Y \rightarrow X$ for each $t \in I$, $h_{1}(U)=Y$, and $h((U \backslash Y) \times[0,1)) \subseteq X \backslash Y$. If, in addition, $h$ can be taken to be nearly stratum preserving, then $Y$ is stratified forward tame in $X$.

Definition 4.1. A space $X$ with a stratification satisfying the Frontier Condition is a homotopically stratified space if the following two conditions are satisfied:

(i) (Forward Tameness) For each $k>i$, the stratum $X_{i}$ is forward tame in $X_{i} \cup X_{k}$.

(ii) (Normal Fibrations) For each $k>i$, the holink evaluation

$$
q: \operatorname{holink}\left(X_{i} \cup X_{k}, X_{i}\right) \rightarrow X_{i}
$$

is a fibration.

The following is the main result from [8] concerning mapping cylinders of stratified approximate fibrations between homotopically stratified spaces. We will use it, along with the embedding result from $\S 3$, in the proof of Theorem 4.3 below.

THEOREM 4.2 [8]. Let $p: X \rightarrow Y$ be a proper map between locally compact homotopically stratified metric spaces each with only finitely many strata, and suppose the strata of $Y$ are path connected. Then $p$ is a stratified approximate fibration if and only if $\operatorname{cyl}(p)$ with the natural partition is a homotopically stratified space.

The teardrop of a stratified approximate fibration. We are now ready for the main result.

TheOREM 4.3. Let $X$ and $Y$ be locally compact, separable, homotopically stratified metric spaces each with only finitely many strata and suppose the strata of $Y$ are path connected. If $p: X \rightarrow Y \times \mathbb{R}$ is a proper stratified 
approximate fibration, then the teardrop $X \cup_{p} Y$ with the natural partition is a homotopically stratified space.

The proof of Theorem 4.3 is based on the following lemmas whose hypotheses include the hypothesis of Theorem 4.3. We also use the notation of $\S 3$. In particular, there is the mapping cylinder collapse $p_{Y \times(-\infty,+\infty]}$ : $\operatorname{cyl}\left(p^{\prime}\right) \rightarrow Y \times(-\infty,+\infty]$.

Lemma 4.4. If $A$ is a closed union of strata of $Y$, then there exists a map

$$
\beta: Y \times(-\infty,+\infty] \rightarrow[0,1]
$$

with $\beta^{-1}(1)=Y \times\{+\infty\}$ such that $A \times(-\infty,+\infty]$ is stratified forward tame in $\operatorname{cyl}\left(p^{\prime}\right)_{\beta}$.

Pr o of. It follows from [7, Theorem 6.3] that $A \times \mathbb{R}$ is stratified forward tame in $Y \times \mathbb{R}$. Thus, Theorem 4.2 above implies that $A \times \mathbb{R}$ is stratified forward tame in $\operatorname{cyl}(p)$. In fact, there is a neighborhood $U$ of $A \times \mathbb{R}$ in $Y \times \mathbb{R}$ and a nearly stratum preserving deformation $G: \operatorname{cyl}\left(p_{U}\right) \times I \rightarrow \operatorname{cyl}(p)$ of $\operatorname{cyl}\left(p_{U}\right)$ to $A \times \mathbb{R}$ in $\operatorname{cyl}(p)$ rel $A \times \mathbb{R}$ where $p_{U}=p \mid: p^{-1}(U) \rightarrow U$ such that $G \mid: U \times I \rightarrow Y \times \mathbb{R}$ is a given nearly stratum preserving deformation of $U$ to $A \times \mathbb{R}$ in $Y \times \mathbb{R}$ rel $A \times \mathbb{R}$ (cf. the proof of [8, Prop. 5.7]). We may assume that $U=V \times \mathbb{R}$ where $V$ is a neighborhood of $A$ in $Y$, that there exists a nearly stratum preserving deformation $g: V \times I \rightarrow Y$ of $V$ to $A$ in $Y$ rel $A$, and that $G \mid U \times I=g \times \operatorname{id}_{\mathbb{R}}$. Let $U_{0}=V \times(-\infty, 0]$ and, for $i=1,2, \ldots$, let $U_{i}=V \times[i-1, i]$. For $i=1,2, \ldots$ choose maps $\delta_{i}: U_{i} \rightarrow(0,1]$ (using a partition of unity) such that if $1-\delta_{i}(p(x)) \leq s \leq 1$ and $x \in p^{-1}\left(U_{i}\right)$, then $G([x, s], t)$ is $1 / i$-close to $G([p(x)], t)$ for each $t \in I$. Let $\delta_{0} \equiv 1$. Consider the subspace $W \subseteq \operatorname{cyl}\left(p^{\prime}\right)$ given by

$$
W=\bigcup_{i=0}^{\infty}\left\{[x, s] \in \operatorname{cyl}(p) \backslash(Y \times \mathbb{R}) \mid x \in p^{-1}\left(U_{i}\right), 1-\delta_{i}(p(x)) \leq s \leq 1\right\}
$$

$$
\cup V \times(-\infty,+\infty] .
$$

Note that $W \cap \operatorname{cyl}(p) \subseteq \operatorname{cyl}\left(p_{U}\right)$ and $W \backslash \operatorname{cyl}(p)=V \times\{+\infty\}$. Now $G \mid(W \cap \operatorname{cyl}(p)) \times I$ extends continuously to $\widetilde{G}: W \times I \rightarrow \operatorname{cyl}\left(p^{\prime}\right)$ by setting $\widetilde{G}(y,+\infty, t)=(g(y, t),+\infty)$ for $y \in V$.

Use a partition of unity to construct a map $\beta: Y \times(-\infty,+\infty] \rightarrow[0,1]$ such that $\beta^{-1}(1)=Y \times\{+\infty\}$ and $\operatorname{cyl}\left(p^{\prime}\right)_{\beta} \cap \operatorname{cyl}\left(p_{U}\right) \subseteq W$. Note that there is a stratum preserving retraction $r: \operatorname{cyl}\left(p^{\prime}\right) \rightarrow \operatorname{cyl}\left(p^{\prime}\right)_{\beta}$ defined by pushing down along the mapping cylinder rays. Since $W \cap \operatorname{cyl}\left(p^{\prime}\right)_{\beta}$ is a neighborhood of $A \times(-\infty,+\infty]$ in $\operatorname{cyl}\left(p^{\prime}\right)_{\beta}$ and there is a nearly stratum preserving deformation

$$
\left(W \cap \operatorname{cyl}\left(p^{\prime}\right)_{\beta}\right) \times I \rightarrow \operatorname{cyl}\left(p^{\prime}\right)_{\beta}, \quad(x, t) \mapsto r \widetilde{G}(x, t),
$$


to $A \times(-\infty,+\infty]$ in $\operatorname{cyl}\left(p^{\prime}\right)_{\beta}$ rel $A \times(-\infty,+\infty]$, we have shown that $A \times$ $(-\infty,+\infty]$ is stratified forward tame in $\operatorname{cyl}\left(p^{\prime}\right)_{\beta}$.

LEMMA 4.5. If $A$ is a closed union of strata of $Y$, then there exists a map

$$
\beta: Y \times(-\infty,+\infty] \rightarrow[0,1]
$$

with $\beta^{-1}(1)=Y \times\{+\infty\}$ such that $A \times\{+\infty\}$ is stratified forward tame in $\operatorname{cyl}\left(p^{\prime}\right)_{\beta}$.

Pr o of. Choose $\beta$ by Lemma 4.4 so that $A \times(-\infty,+\infty]$ is stratified forward tame in $\operatorname{cyl}\left(p^{\prime}\right)_{\beta}$. Let $U$ be a neighborhood of $A \times(-\infty,+\infty]$ in $\operatorname{cyl}\left(p^{\prime}\right)_{\beta}$ for which there is a nearly stratum preserving deformation $h: U \times I \rightarrow$ $\operatorname{cyl}\left(p^{\prime}\right)_{\beta}$ of $U$ to $A \times(-\infty,+\infty]$ in $\operatorname{cyl}\left(p^{\prime}\right)_{\beta}$ rel $A \times(-\infty,+\infty]$. By Proposition 3.2 there is a nearly stratum preserving deformation $g: \operatorname{cyl}\left(p^{\prime}\right)_{\beta} \times I \rightarrow$ $\operatorname{cyl}\left(p^{\prime}\right)_{\beta}$ to $Y \times\{+\infty\}$ rel $Y \times\{+\infty\}$. Define $H: U \times I \rightarrow \operatorname{cyl}\left(p^{\prime}\right)_{\beta}$ by $H(x, t)=g(h(x, t), t)$. It is not difficult to see that $H$ is a nearly stratum preserving deformation of $U$ to $Y \times\{+\infty\}$ in $\operatorname{cyl}\left(p^{\prime}\right)_{\beta}$ rel $Y \times\{+\infty\}$. Perhaps the only point that needs to be addressed is why $H_{1}(U) \subseteq A \times\{+\infty\}$. To this end, let $x \in U$. If $h_{1}(x) \in A \times(-\infty,+\infty]$, then clearly $H_{1}(x) \in$ $A \times\{+\infty\}$. If $h_{1}(x) \in A \times \mathbb{R}$, then $g$ being nearly stratum preserving implies $g(h(x, 1), 1) \in \operatorname{cl}(A \times \mathbb{R})=A \times(-\infty,+\infty]$. Also, $g(h(x, 1), 1) \in Y \times\{+\infty\}$. Since $Y \times\{+\infty\} \cap A \times(-\infty,+\infty]=A \times\{+\infty\}$, we are done.

Lemma 4.6. If $A$ is a closed union of strata of $Y$, then $A$ is stratified forward tame in $X \cup_{p} Y$.

Pr o o f. By Lemma 4.5 there are a map $\beta$ and a neighborhood $U$ of $A \times$ $\{+\infty\}$ in $\operatorname{cyl}\left(p^{\prime}\right)_{\beta}$ for which there is a nearly stratum preserving deformation $H: U \times I \rightarrow \operatorname{cyl}\left(p^{\prime}\right)_{\beta}$ of $U$ to $Y \times\{+\infty\}$ in $\operatorname{cyl}\left(p^{\prime}\right)_{\beta}$ rel $Y \times\{+\infty\}$. Let $\alpha_{\beta}$ : $X \cup_{p} Y \rightarrow \operatorname{cyl}\left(p^{\prime}\right)_{\beta}$ be the embedding from $\S 3$. Define a stratum preserving retraction

$r: \operatorname{cyl}\left(p^{\prime}\right)_{\beta} \backslash(Y \times \mathbb{R}) \rightarrow \alpha_{\beta}\left(X \cup_{p} Y\right), \quad \begin{cases}r([x, t])=[x, \beta(p(x))] & \text { if } x \in X, \\ r([y,+\infty])=[y,+\infty] & \text { if } y \in Y .\end{cases}$

Then $h: \alpha_{\beta}^{-1}(U) \times I \rightarrow X \cup_{p} Y$ defined by $h(x, t)=\alpha_{\beta}^{-1}\left(r h\left(\alpha_{\beta}(x), t\right)\right)$ is a nearly stratum preserving deformation of $\alpha_{\beta}^{-1}(U)$ to $A$ in $X \cup_{p} Y$ rel $A$.

LEMma 4.7. The natural partition of $X \cup_{p} Y$ is a stratification satisfying the Frontier Condition and the Forward Tameness condition 4.1(i).

Proof. The partition of $X \cup_{p} Y$ is obviously locally finite (in fact, it is finite). That each stratum is locally closed (i.e., the intersection of an open set and a closed set) follows easily from the fact that $Y$ is closed in $X \cup_{p} Y$. For both the Frontier Condition and Forward Tameness there is only one 
nontrivial case to consider: suppose $X_{i}, Y_{j}$ are strata of $X, Y$, respectively, and $Y_{j} \cap \operatorname{cl} X_{i} \neq \emptyset$ where the closure is taken in $X \cup_{p} Y$.

For the Frontier Condition we need to show that $Y_{j} \subseteq \operatorname{cl} X_{i}$. We begin by showing that $\left(Y_{j} \times \mathbb{R}\right) \cap \operatorname{cl}\left(X_{i} \times[0,1)\right) \neq \emptyset$ in $\operatorname{cyl}(p)$. Choose $y_{0} \in Y_{j} \cap \operatorname{cl} X_{i}$ so that there is a sequence $\left\{x_{n}\right\}$ in $X_{i}$ with $x_{n} \rightarrow y_{0}$ in $X \cup_{p} Y$. It follows that $p\left(x_{n}\right) \rightarrow\left(y_{0},+\infty\right)$ in $Y \times(-\infty,+\infty]$. For each $n$, write $p\left(x_{n}\right)=$ $\left(x_{n}^{1}, x_{n}^{2}\right) \in Y \times \mathbb{R}$. Let $\omega_{n}: I \rightarrow Y \times \mathbb{R}$ be the path $\omega_{n}(t)=\left(x_{n}^{1},(1-t) x_{n}^{2}\right)$, so that it is a stratum preserving path from $p\left(x_{n}\right)$ to $\left(x_{n}^{1}, 0\right)$. Since $p$ is a stratified approximate fibration, there are stratum preserving approximate lifts $\widetilde{\omega}_{n}: I \rightarrow X$ with $\widetilde{\omega}_{n}(0)=x_{n}$. Thus, $\widetilde{\omega}_{n}(1) \in X_{i}$ and $p$, $\widetilde{\omega}_{n}(1) \rightarrow\left(y_{0}, 0\right) \in Y_{j} \times \mathbb{R}$, showing that $\left(Y_{j} \times \mathbb{R}\right) \cap \operatorname{cl}\left(X_{i} \times[0,1)\right) \neq \emptyset$ in $\operatorname{cyl}(p)$ as promised. Now [8] implies that $\operatorname{cyl}(p)$ satisfies the Frontier Condition so that $Y_{j} \times \mathbb{R} \subseteq \operatorname{cl}\left(X_{i} \times[0,1)\right)$ in $\operatorname{cyl}(p)$. In particular, if $y_{0} \in Y_{j}$, then $\left\{y_{0}\right\} \times \mathbb{R} \subseteq \operatorname{cl}\left(X_{i} \times[0,1)\right)$. Thus, for each $n=1,2, \ldots$ there exists $\left(x_{n}, t_{n}\right) \in X_{i} \times[0,1)$ such that $\left(x_{n}, t_{n}\right)$ is so close to $\left(y_{0}, n\right)$ that $p\left(x_{n}\right)$ is $1 / n$-close to $\left(y_{0}, n\right)$ in $Y \times \mathbb{R}$. It follows that $x_{n} \rightarrow y_{0}$ in $X \cup_{p} Y$, verifying the Frontier Condition.

To show that $Y_{j}$ is forward tame in $X_{i} \cup Y_{j}$, we know from Lemma 4.6 that $Y^{j}=\operatorname{cl} Y_{j}$ is stratified forward tame in $X \cup_{p} Y$. So let $U$ be a neighborhood of $Y^{j}$ in $X \cup_{p} Y$ for which there is a nearly stratum preserving deformation $h: U \times I \rightarrow X \cup_{p} Y$ of $U$ to $Y^{j}$ in $X \cup_{p} Y$ rel $Y^{j}$. Let $V=h_{1}^{-1}\left(Y_{j}\right) \cap\left[X_{i} \cup Y_{j}\right]$. Then $V$ is a neighborhood of $Y_{j}$ in $X_{i} \cup Y_{j} \subseteq X \cup_{p} Y$ and $h$ restricts to a nearly stratum preserving deformation of $V$ to $Y_{j}$ in $X_{i} \cup Y_{j}$ rel $Y_{j}$.

Proof of Theorem 4.3. Given Lemma 4.7 it only remains to verify the Normal Fibrations condition 4.1(ii). As in the proof of Lemma 4.7, there is only one nontrivial case to consider: suppose $X_{i}, Y_{j}$ are strata of $X, Y$, respectively, and $Y_{j} \cap \operatorname{cl} X_{i} \neq \emptyset$ where the closure is taken in $X \cup_{p} Y$ (hence, $Y_{j} \subseteq \operatorname{cl} X_{i}$ ). We need to show that the holink evaluation $\operatorname{holink}\left(X_{i} \cup Y_{j}, Y_{j}\right) \rightarrow Y_{j}$ is a fibration.

Let $U$ be a neighborhood of $Y^{j}=\operatorname{cl} Y_{j}$ in $Y$ for which there is a nearly stratum preserving deformation $h: U \times I \rightarrow Y$ of $U$ to $Y^{j}$ in $Y$ rel $Y^{j}$ [7, Theorem 6.3]. Let $p_{U \times \mathbb{R}}=p \mid p^{-1}(U \times \mathbb{R}) \rightarrow U \times \mathbb{R}$. According to $[8,5.6]$ there exists a homotopy $H: \operatorname{cyl}\left(p_{U \times \mathbb{R}}\right) \times I \rightarrow \operatorname{cyl}(p)$ such that

(1) $H_{0}=$ inclusion,

(2) $H \mid U \times \mathbb{R} \times I=h \times \operatorname{id}_{\mathbb{R}}$,

(3) $H\left(\left(\operatorname{cyl}\left(p_{U \times \mathbb{R}}\right) \backslash U \times \mathbb{R}\right) \times I\right) \subseteq \operatorname{cyl}(p) \backslash(Y \times \mathbb{R})$,

(4) $H \mid:\left(\operatorname{cyl}\left(p_{U \times \mathbb{R}}\right) \backslash U \times \mathbb{R}\right) \times I \rightarrow \operatorname{cyl}(p)$ is a stratum preserving homotopy,

(5) $H$ is level preserving, i.e., $p_{I} \circ H_{t}=p_{I}$ for each $t \in I$ (this is not stated in [8], but follows from the proof). 
A partition of unity argument shows that there exists a map $\beta: Y \times$ $(-\infty,+\infty] \rightarrow I$ such that $\beta^{-1}(1)=Y \times\{+\infty\}$ and $H \mid: \operatorname{cyl}\left(p_{U \times \mathbb{R}}\right)_{\beta} \times I$ $\rightarrow \operatorname{cyl}(p)$ extends continuously to $U \times\{+\infty\} \times I$ via $h \times \operatorname{id}_{\{+\infty}$. Let $p_{U \times \mathbb{R}}^{\prime}$ be the composition

$$
p_{U \times \mathbb{R}}^{\prime}: p^{-1}(U \times \mathbb{R}) \stackrel{p_{U \times \mathbb{R}}}{\longrightarrow} U \times \mathbb{R} \stackrel{\subseteq}{\longrightarrow} U \times(-\infty,+\infty] .
$$

Define $\widetilde{H}: \operatorname{cyl}\left(p_{U \times \mathbb{R}}^{\prime}\right)_{\beta} \times I \rightarrow \operatorname{cyl}\left(p^{\prime}\right)_{\beta}$ by $\widetilde{H} \mid \operatorname{cyl}\left(p_{U \times \mathbb{R}}\right)_{\beta} \times I=\varrho \circ H$ and $\widetilde{H}_{t} \mid U \times\{+\infty\}=h_{t} \times \operatorname{id}_{\{+\infty\}}$ for each $t \in I$, where $\varrho: \operatorname{cyl}(p) \rightarrow \operatorname{cyl}\left(p^{\prime}\right)_{\beta}$ is the natural retraction obtained by pushing down the mapping cylinder rays.

Let $U^{\prime}=U \backslash h_{1}^{-1}\left(Y^{j} \backslash Y_{j}\right)$. Note that $U^{\prime}$ is a neighborhood of $Y_{j}$ in $Y$ and $h: U^{\prime} \times I \rightarrow Y \backslash\left(Y^{j} \backslash Y_{j}\right)$. Thus, $p^{-1}\left(U^{\prime} \times \mathbb{R}\right) \cup U^{\prime}$ is a neighborhood of $Y_{j}$ in $X \cup_{p} Y$. It follows that if $X_{i}^{\prime}=X_{i} \cap p^{-1}\left(U^{\prime} \times \mathbb{R}\right)$, then it suffices to show that holink $\left(X_{i}^{\prime} \cup Y_{j}, Y_{j}\right) \rightarrow Y_{j}$ is a fibration. Let $Y_{j}^{\prime}=Y_{j} \times\{+\infty\}$ and $X_{i}^{\prime \prime}=X_{i}^{\prime} \times[0,1) \cap \operatorname{cyl}\left(p_{U \times \mathbb{R}}^{\prime}\right)_{\beta}$. Recall from 3.1 that $\alpha_{\beta}: X \cup_{p} Y \rightarrow \operatorname{cyl}\left(p^{\prime}\right)_{\beta}$ is an embedding. Now note that this embedding restricts to an embedding $\alpha_{\beta} \mid: X_{i}^{\prime} \cup Y_{j} \rightarrow X_{i}^{\prime \prime} \cup Y_{j}^{\prime} \subseteq \operatorname{cyl}\left(p_{U \times \mathbb{R}}^{\prime}\right)_{\beta}$. Moreover, the image $\alpha_{\beta}\left(X_{i}^{\prime} \cup Y_{j}\right)$ is a stratum preserving strong deformation retract of $X_{i}^{\prime \prime} \cup Y_{j}^{\prime}$ rel $Y_{j}^{\prime}=\alpha_{\beta}\left(Y_{j}\right)$. This implies that the holink evaluation $\operatorname{holink}\left(X_{i}^{\prime} \cup Y_{j}, Y_{j}\right) \rightarrow Y_{j}$ is a fiber preserving retract of $\operatorname{holink}\left(X_{i}^{\prime \prime} \cup Y_{j}^{\prime}, Y_{j}^{\prime}\right) \rightarrow Y_{j}^{\prime}$. Thus, it suffices to prove that $q: \operatorname{holink}\left(X_{i}^{\prime \prime} \cup Y_{j}^{\prime}, Y_{j}^{\prime}\right) \rightarrow Y_{j}^{\prime}$ is a fibration. To this end, consider a lifting problem:

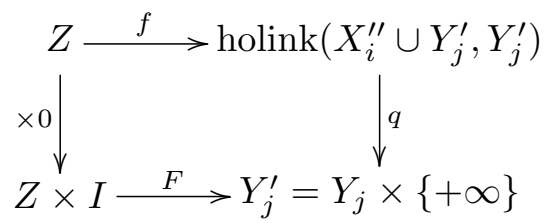

Now $f$ induces a map $f^{\prime}: Z \times(0,1] \rightarrow \operatorname{holink}\left(X_{i}^{\prime \prime} \cup\left(U^{\prime} \times \mathbb{R}\right), U^{\prime} \times \mathbb{R}\right)$ by letting $f^{\prime}(z, s)$ be the path beginning in $U^{\prime} \times \mathbb{R}$ and continuing up the mapping cylinder ray until reaching $f(z)(s)$; that is,

$$
f^{\prime}(z, s)(t)=\left[p_{X} f(z)(s), t \cdot p_{I} f(z)(s)+(1-t)\right] \in \operatorname{cyl}(p)
$$

(where we are using the notation from $\S 2$ ).

Use $H$ to deform $f^{\prime}$ to a new map $f^{\prime \prime}$ by defining

$$
f^{\prime \prime}(z, s)(t)=H\left(f^{\prime}(z, s)(t), 1-t\right)
$$

for $(z, s, t) \in Z \times(0,1] \times I$. Now $f^{\prime \prime}(z, s)$ is still a path in $\operatorname{cyl}\left(p^{\prime}\right)$ which terminates at $f(z)(s)$, but it originates at the point $\left(h_{1} \times \operatorname{id}_{\mathbb{R}}\right)\left(p_{Y \times \mathbb{R}} f(z)(s)\right) \in$ $Y_{j} \times \mathbb{R}$. Thus, $f^{\prime \prime}: Z \times(0,1] \rightarrow \operatorname{holink}\left(\left(X_{i} \times[0,1)\right) \cup\left(Y_{j} \times \mathbb{R}\right), Y_{j} \times \mathbb{R}\right)$. 
We will now define a map $F^{\prime}: Z \times(0,1] \times I \rightarrow Y_{j} \times \mathbb{R}$ so that there is a commuting diagram

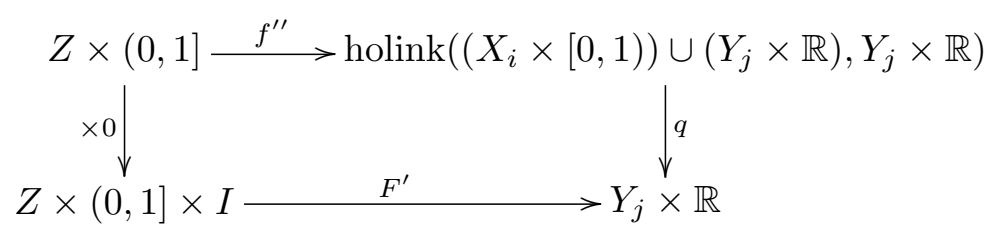

We will use the natural map $p_{Y \times \mathbb{R}}: \operatorname{cyl}(p) \rightarrow Y \times \mathbb{R}$ along with the projections $\pi_{Y}: Y \times \mathbb{R} \rightarrow Y$ and $\pi_{\mathbb{R}}: Y \times \mathbb{R} \rightarrow \mathbb{R}$. Define $F^{\prime}$ by

$$
\begin{aligned}
& \pi_{\mathbb{R}} F^{\prime}(z, s, t)=\pi_{\mathbb{R}} p_{Y \times \mathbb{R}}\left(f^{\prime \prime}(z, s)(0)\right), \\
& \pi_{Y} F^{\prime}(z, s, t)= \begin{cases}\pi_{Y} f^{\prime \prime}(z, s-t)(0) & \text { if } 0 \leq t<s, \\
\pi_{Y} F(z, 0) & \text { if } t=s, \\
\pi_{Y} F(z,(t-s) /(1-s)) & \text { if } s<t \leq 1 .\end{cases}
\end{aligned}
$$

Since $p: X \rightarrow Y \times \mathbb{R}$ is a stratified approximate fibration, it follows that $\operatorname{cyl}(p)$ is a homotopically stratified space (Theorem 4.2). Hence, (4.3.2) has a solution $\widetilde{F}: Z \times(0,1] \times I \rightarrow \operatorname{holink}\left(\left(X_{i} \times[0,1)\right) \cup\left(Y_{j} \times \mathbb{R}\right), Y_{j} \times \mathbb{R}\right)$.

In fact, $F^{\prime}$ extends continuously to

$$
F^{\prime \prime}: Z \times I \times I \rightarrow \operatorname{cyl}\left(p^{\prime}\right)
$$

by setting $F^{\prime \prime}(z, 0, t)=F(z, t)$. According to [7, Lemma 8.1] there exists a map $u: Z \times I \times I \rightarrow I$ such that $u^{-1}(0)=Z \times I \times\{0\}$ and such that the function $F^{*}: Z \times I \rightarrow \operatorname{cyl}\left(p^{\prime}\right)^{I}$ defined by

$$
F^{*}(z, s)(t)= \begin{cases}\varrho \widetilde{F}(z, t, s)(u(z, s, t)) & \text { if } t>0 \\ F(z, s) & \text { if } t=0\end{cases}
$$

is continuous. Inspection shows that $F^{*}$ is a solution of (4.3.1).

5. Compactly dominated local holinks. In this section we embellish Theorem 4.3 by considering homotopically stratified spaces whose homotopy links have an additional finite domination property: compactly dominated local homotopy links. Theorem 5.4 below shows that the teardrop will also have this property. This is an important fact because the domination property is one of the defining properties of a manifold stratified space (see $\S 6$ ).

To motivate the definition, recall that a $\mathrm{CW}$ complex $W$ is dominated by a finite CW complex if and only if $W$ is dominated by a compact subspace. In the current stratified setting, we find the formulation with compact subspaces to be more convenient.

Let $X$ be a space with a partition $\left\{X_{i}\right\}_{i \in \mathcal{I}}$. Let $x_{0} \in X_{i} \subseteq X$. The local holink at $x_{0}$ is 


$$
\operatorname{holink}\left(X, x_{0}\right)=\left\{\omega \in \operatorname{holink}_{\mathrm{s}}\left(X, X_{i}\right) \mid \omega(0)=x_{0}\right\} .
$$

The local holink inherits a natural partition from $\operatorname{holink}_{\mathrm{s}}\left(X, X_{i}\right)$.

Definition 5.1. $X$ has a compactly dominated local holink at $x_{0}$ if there exist a compact subset $C \subseteq \operatorname{holink}\left(X, x_{0}\right)$ and a stratum preserving deformation

$$
d: \operatorname{holink}\left(X, x_{0}\right) \times I \rightarrow \operatorname{holink}\left(X, x_{0}\right)
$$

such that $d_{1}\left(\operatorname{holink}\left(X, x_{0}\right)\right) \subseteq C$. We say $X$ has compactly dominated local holinks if for every $x_{0} \in X, X$ has a compactly dominated local holink at $x_{0}$.

LEMMA 5.2. Let $X$ be a homotopically stratified metric space with only finitely many strata and suppose the strata are ANRs. If $x_{0} \in X$, then there exist a neighborhood $U$ of $x_{0}$ and a nearly stratum preserving deformation $h: U \times I \rightarrow X$ of $U$ to $\left\{x_{0}\right\}$ in $X$ rel $\left\{x_{0}\right\}$.

Proof. Let $X_{i}$ be the stratum containing $x_{0}$. Since $X_{i}$ is an ANR, there exist an arbitrarily small neighborhood $V$ of $x_{0}$ in $X_{i}$ and a deformation $g: V \times I \rightarrow X_{i}$ of $V$ to $x_{0}$ in $X_{i}$ rel $x_{0}$. The Homotopy Extension Property gives an extension of $g$ to a deformation $\widetilde{g}: X_{i} \times I \rightarrow X_{i}$ which is supported on a slightly larger neighborhood of $x_{0}$ in $X_{i}$. Thus, $\widetilde{g}$ extends via the identity to a stratum preserving deformation with the same name $\widetilde{g}: X^{i} \times I \rightarrow$ $X^{i}$. Moreover, the Stratum Preserving Deformation Extension Property [7] implies that $\widetilde{g}$ can be extended to a stratum preserving deformation $\widetilde{g}$ : $X \times I \rightarrow X$, also with the same name. Since $X^{i}$ is stratified forward tame in $X$ [7, Theorem 6.3], there exist a neighborhood $W$ of $X^{i}$ in $X$ and a nearly stratum preserving deformation $k: W \times I \rightarrow X$ of $W$ to $X^{i}$ in $X$ rel $X^{i}$. Now define $h^{\prime}: W \times I \rightarrow X$ by $h^{\prime}(x, t)=\widetilde{g}_{t}\left(k_{t}(x)\right)$. Finally, let $U=k_{1}^{-1}(V)$ and $h=h^{\prime} \mid U \times I$.

Lemma 5.3. Let $X$ be a homotopically stratified metric space with only finitely many strata and suppose the strata are ANRs. Then $X$ has a compactly dominated local holink at $x_{0} \in X_{i} \subseteq X$ if and only if for every neighborhood $U$ of $x_{0}$ there exist a neighborhood $V$ of $x_{0}$ with $V \subseteq U$, a compact subset $K \subseteq U$, and a stratum preserving deformation $g:\left(V \backslash X_{i}\right) \times I \rightarrow U$ such that $g_{1}\left(V \backslash X_{i}\right) \subseteq K$.

Pr o of. Suppose first that $X$ has a compactly dominated local holink at $x_{0}$ and let $U$ be a given neighborhood. An element of holink $\left(X, x_{0}\right)$ is a nearly stratum preserving path in $X$ beginning at $x_{0}$. It can be shrunk along itself to give a nearly stratum preserving path in $U$ beginning at $x_{0}$. A partition of unity argument turns this idea into a stratum preserving deformation of $\operatorname{holink}\left(X, x_{0}\right)$ to $\operatorname{holink}\left(U, x_{0}\right) \operatorname{rel} \operatorname{holink}\left(U, x_{0}\right)$. Hence, $\operatorname{holink}\left(U, x_{0}\right)$ is compactly dominated; i.e., there exist a compact subset $C \subseteq \operatorname{holink}\left(U, x_{0}\right)$ and a stratum preserving deformation $d: \operatorname{holink}\left(U, x_{0}\right) \times I \rightarrow \operatorname{holink}\left(U, x_{0}\right)$ 
such that $d_{1}\left(\operatorname{holink}\left(U, x_{0}\right)\right) \subseteq C$. Use Lemma 5.2 to get a neighborhood $V$ of $x_{0}$ and a nearly stratum preserving deformation $h: V \times I \rightarrow U$ of $V$ to $\left\{x_{0}\right\}$ in $U$ rel $x_{0}$. Let $\widetilde{h}: V \rightarrow \operatorname{holink}\left(U, x_{0}\right)$ be given by $\widetilde{h}(x)(t)=h(x, 1-t)$. Let $p: \operatorname{holink}\left(U, x_{0}\right) \rightarrow U$ be evaluation at $1(p(\omega)=\omega(1))$ and let $K=p(C)$, a compact subset of $U$. Define $g:\left(V \backslash X_{i}\right) \times I \rightarrow U$ by $g(x, t)=d(\widetilde{h}(x), t)(1)$, which is a deformation satisfying the desired properties.

Conversely, let $U$ be a neighborhood of $x_{0}$ for which there exists a nearly stratum preserving deformation $h: U \times I \rightarrow X$ of $U$ to $x_{0}$ in $X$ rel $x_{0}$ (Lemma 5.2). Then the hypothesis implies that there exist a neighborhood $V$ of $x_{0}, V \subseteq U$, a compact subset $K \subseteq U$, and a stratum preserving deformation $g:\left(V \backslash X_{i}\right) \times I \rightarrow U$ such that $g_{1}\left(V \backslash X_{i}\right) \subseteq K$. Define $\widetilde{h}: K \rightarrow \operatorname{holink}\left(X, x_{0}\right)$ by $\widetilde{h}(x)(t)=h(x, 1-t)$. Let $C=\widetilde{h}(K)$, a compact subset of $\operatorname{holink}\left(X, x_{0}\right)$. We will show that there is a stratum preserving deformation of holink $\left(X, x_{0}\right)$ into $C$. First, note that by the argument in the first part, there is a stratum preserving deformation of $\operatorname{holink}\left(X, x_{0}\right)$ into holink $\left(V, x_{0}\right)$, Second, there is a stratum preserving deformation $f: \operatorname{holink}\left(V, x_{0}\right) \times I \rightarrow \operatorname{holink}\left(U, x_{0}\right)$ of $\operatorname{holink}\left(V, x_{0}\right)$ into $\mathcal{K}$, where $\mathcal{K}=$ $\left\{\omega \in \operatorname{holink}\left(U, x_{0}\right) \mid \omega(1) \in K\right\}$. This is defined by

$$
f(\omega, t)(s)= \begin{cases}\omega(2 s /(2-t)) & \text { if } 0 \leq s \leq(2-t) / 2 \\ g(\omega(1), 2 s+t-2) & \text { if }(2-t) / 2 \leq s \leq 1\end{cases}
$$

In words, $\omega$ is stretched out using $g$ to a path terminating in $K$. Finally, there is a stratum preserving deformation $e: \mathcal{K} \times I \rightarrow \operatorname{holink}\left(X, x_{0}\right)$ of $\mathcal{K}$ into $C$ defined by

$$
e(\omega, t)(s)=h(\omega((1-s) t+s),(1-s) t)
$$

TheOREM 5.4. Let $X$ and $Y$ be locally compact, separable, homotopically stratified metric spaces with only finitely many strata. Suppose the strata are ANRs and $X$ and $Y$ have compactly dominated local holinks. If $p: X \rightarrow$ $Y \times \mathbb{R}$ is a proper stratified approximate fibration, then $X \cup_{p} Y$ with the natural partition has compactly dominated local holinks. Moreover, $X \cup_{p} Y$ is a locally compact, separable homotopically stratified metric space with only finitely many ANR strata.

Proof. We only need to show that $X \cup_{p} Y$ has compactly dominated local holinks at points of $Y$. Thus, let $y \in Y$, say $y \in Y_{j}$. We will use Lemma 5.3. Neighborhoods of $y$ in $X \cup_{p} Y$ contain neighborhoods of the form $U=c^{-1}(W \times[N,+\infty])$ where $N \geq 0, W$ is a compact neighborhood of $y$ in $Y$, and $c: X \cup_{p} Y \rightarrow Y \times(-\infty,+\infty]$ is the teardrop collapse. Suppose one such neighborhood is given. Since $Y$ has compactly dominated local holinks, Lemma 5.3 implies that there exist an open neighborhood $W_{1}$ of $y$ in $Y$ and a stratum preserving deformation $g:\left(W_{1} \backslash Y_{j}\right) \times I \rightarrow W$ such that $g_{1}\left(W_{1} \backslash Y_{j}\right) \subseteq W \backslash W_{1}$ (which is compact). 
Now $W_{1} \backslash Y^{j}$ is open in $Y$, so there exists an open subset $\widetilde{W}_{1} \subseteq X \cup_{p} Y$ such that $\widetilde{W}_{1} \cap Y=W_{1} \backslash Y^{j}$ and $\mathrm{cl} \widetilde{W}_{1} \subseteq U$. The Stratum Preserving Deformation Extension Property of [7] implies that there is an extension of $g$ to a stratum preserving deformation $\widetilde{g}: \widetilde{W}_{1} \times I \rightarrow U$. (Actually, [7, Corollary 6.4] does not apply exactly as stated, but its proof does.)

Let $W_{2}$ be a compact neighborhood of $y$ in $Y$ such that $W_{2} \subseteq W_{1}$. Let $\varrho: W \backslash Y^{j} \rightarrow I$ be a map such that $\varrho^{-1}(0)=U \backslash\left(\widetilde{W}_{1} \cup Y^{j}\right)$ and $\varrho^{-1}(1)=W_{2} \backslash Y^{j}$. Then $\widetilde{g}$ can be modified to $\widehat{g}:\left(U \backslash Y^{j}\right) \times I \rightarrow U$ by

$$
\widehat{g}(x, t)= \begin{cases}x & \text { if } x \in U \backslash\left(\widetilde{W}_{1} \cup Y^{j}\right), t \in I, \\ \widetilde{g}(x, \varrho(x) t) & \text { otherwise. }\end{cases}
$$

In particular, $\widehat{g} \mid\left(W_{1} \backslash Y^{j}\right) \times I=g$.

Use the fact that $p: X \rightarrow Y \times \mathbb{R}$ is a stratified approximate fibration to get a stratum preserving deformation $h: X \times I \rightarrow X$ such that

$$
h_{1}\left(p^{-1}\left(W_{2} \times[N+1,+\infty)\right)\right) \subseteq p^{-1}(W \times[N, N+2]) .
$$

Then define $H:\left(U \backslash Y^{j}\right) \times I \rightarrow X \cup_{p} Y$ by

$$
H(x, t)= \begin{cases}\widehat{g}(x, 2 t) & \text { if } 0 \leq t \leq 1 / 2, \\ h(\widehat{g}(x, 1), 2 t-1) & \text { if } 1 / 2 \leq t \leq 1\end{cases}
$$

Note that $H_{1}\left(c^{-1}\left(W_{2} \times[N+1,+\infty] \backslash Y^{j}\right)\right)$ is a compact subset of $U$. Thus, Lemma 5.3 implies that $X \cup_{p} Y$ has a compactly dominated local holink at $x_{0}$.

The additional properties listed in Theorem 5.4 are either obvious or follow from Theorem 4.3, from [11, Lemma 3.15], or from the proof of [11, Corollary 4.10].

6. Manifold stratified teardrops. In this section we apply the previous results to teardrops of manifold stratified approximate fibrations. A homotopically stratified space $X$ with compactly dominated local holinks is a manifold stratified space if, in addition, $X$ is a locally compact, separable metric space and each stratum is a topological manifold (without boundary). A proper stratified approximate fibration $p: X \rightarrow Y$ between manifold stratified spaces is called a manifold stratified approximate fibration.

THEOREM 6.1. If $X$ and $Y$ are manifold stratified spaces each with only finitely many strata and $p: X \rightarrow Y \times \mathbb{R}$ is a manifold stratified approximate fibration, then the teardrop $X \cup_{p} Y$ with the natural partition is a manifold stratified space.

Proof. Immediate from Theorem 5.4.

REMARK 6.2. (1) If $X$ and $Y$ are manifolds (i.e., manifold stratified spaces with exactly one stratum each), then a manifold stratified approx- 
imate fibration $p: X \rightarrow Y \times \mathbb{R}$ is called simply a manifold approximate fibration. In this case, $X \cup_{p} Y$ has compactly dominated local holinks (in fact, finitely dominated) by [11, Corollary 4.10]. It follows that Theorem 6.1 reduces to [11, Corollary 4.11] in this special case.

(2) Theorem 6.1 was announced in [6, Theorem 3.1] with the compactly dominated local holinks condition replaced by an apparently stronger condition (called "finite domination" in [6]). The relationship between these conditions and the reverse tameness condition of Quinn will be clarified in a future paper. For current information, see [15, Proposition 2.15 and Lemma 4.6] and [10, Chapters 8, 9].

7. Examples. In this section we try to give enough examples of manifold stratified approximate fibrations to show that there are plenty of opportunities to perform the teardrop construction and apply the results of this paper. Hopefully, these examples foreshadow future applications.

7.1. Manifold approximate fibrations. We begin with manifold approximate fibrations. For more complete details and references, see [6], [11], [12], [13], [14].

(1) Locally flat submanifolds of topological manifolds (of high dimension) have manifold approximate fibration mapping cylinder neighborhoods [4], [13]. These neighborhoods need not be fiber bundle mapping cylinders [16].

(2) A noncompact manifold $M$ (of high dimension) with one end which is tame in the sense of Siebenmann [17] has a neighborhood $U$ of the end equipped with a manifold approximate fibration $p: U \rightarrow \mathbb{R}$ (see [10]). In this case, the one-point compactification $M \cup\{\infty\}$ of $M$ is a manifold stratified space with two strata, $M$ and $\{\infty\}$. The teardrop $U \cup_{p}\{\infty\}$ is a neighborhood of the singular point. $M$ has a manifold completion if and only if the singular point has a mapping cylinder neighborhood.

(3) More generally, if $(M, B)$ is a manifold stratified pair (i.e., $M$ is a manifold stratified space with two strata, $M \backslash B$ and $B$ ), then $B$ has a neighborhood $U$ for which there is a manifold approximate fibration $p: U \backslash B \rightarrow$ $B \times \mathbb{R}$ and $U$ is homeomorphic to the teardrop $(U \backslash B) \cup_{p} B$ rel $B$ (see [11]). As in (2), $B$ need not have a mapping cylinder neighborhood; even when it does, the neighborhood need not be a fiber bundle mapping cylinder. Examples are constructed in [11] with $B=S^{1}$ which have manifold approximate fibration mapping cylinder neighborhoods, but not fiber bundle mapping cylinder neighborhoods, even after arbitrary euclidean stabilization.

(4) Manifold approximate fibrations arise naturally in controlled topology. If $p: M \rightarrow B$ is a fiber bundle between closed manifolds, $f: N \rightarrow M$ is a homotopy equivalence with $N$ also a closed manifold, then $f$ is a controlled homotopy equivalence if and only if $p f: N \rightarrow B$ is a manifold approximate 
fibration [2], [5]. The same result holds if $p$ is only required to be a manifold approximate fibration.

7.2. Manifold stratified approximate fibrations. A generalization of 7.1(2) above to the multiple strata case has been announced [6]. Here are some other examples.

(1) Let $G$ be a finite group acting locally linearly on a manifold $M$. With the natural orbit type stratification, $M$ and $M / G$ are manifold stratified spaces (in fact, locally conelike) and Beshears [1] has shown that the orbit map $M \rightarrow M / G$ is a manifold stratified approximate fibration (even a stratified fibration). He also extends this result to so-called Quinn actions.

(2) If $p: X \rightarrow Y$ is a proper algebraic map between algebraic varieties, then $X$ and $Y$ have natural Whitney stratifications (in particular, they are manifold stratified spaces) for which $p$ is a manifold stratified approximate fibration [8].

7.3. Teardrop collapse. Teardrops can be used to construct new stratified approximate fibrations from other ones.

Proposition 7.1. Let $X$ and $Y$ be locally compact homotopically stratified metric spaces. If $p: X \rightarrow Y \times \mathbb{R}$ is a proper stratified approximate fibration, then the teardrop collapse $c: X \cup_{p} Y \rightarrow Y \times(-\infty,+\infty]$ is also a proper stratified approximate fibration.

Pr o of. For a stratified lifting problem

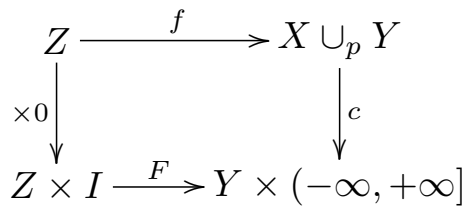

let $Z_{0}=f^{-1}(Y)$ and $Z_{1}=f^{-1}(X)$. According to [7, Remark 5.5(2)] we may assume that $Z$ is a metric space. Restriction gives another stratified lifting problem:

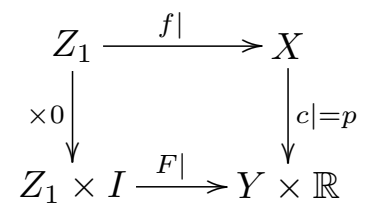

Let

$$
G: Z_{1} \times I \times[0,1) \rightarrow X
$$

be a stratified controlled solution of this second problem. In particular,

$$
\bar{G}: Z_{1} \times I \times I \rightarrow Y \times \mathbb{R}
$$


defined by $\bar{G} \mid Z_{1} \times I \times[0,1)=p G$ and $\bar{G}(z, s, 1)=F(z, s)$ for each $(z, s) \in$ $Z_{1} \times I$, is continuous. Extend $G$ to

$$
G^{+}:\left(Z_{1} \times I \times[0,1)\right) \cup\left(Z_{0} \times I \times\{1\}\right) \rightarrow X \cup_{p} Y
$$

by setting $G^{+}(z, s, 1)=\pi_{Y} F(z, s)$ for each $(z, s) \in Z_{0} \times I$, where

$$
\pi_{Y}: Y \times(-\infty,+\infty] \rightarrow Y
$$

is projection. Now $G^{+}$is not continuous, but the function

$$
\bar{G}^{+}:\left(Z_{1} \times I \times I\right) \cup\left(Z_{0} \times I \times\{1\}\right) \rightarrow Y \times(-\infty,+\infty]
$$

defined by $\bar{G}^{+}=c G^{+}$, i.e.,

$$
\left\{\begin{array}{l}
\bar{G}^{+} \mid\left(Z_{1} \times I \times I\right)=\bar{G}, \quad \text { and } \\
\bar{G}^{+}(z, s, 1)=F(z, s) \quad \text { if }(z, s) \in Z_{0} \times I
\end{array}\right.
$$

is continuous when restricted to $Z_{1} \times I \times I$ and to $Z \times I \times\{1\}$. It follows from a partition of unity argument (this is where the metrizability of $Z$ is used, cf. [7, Lemma 8.1]) that there is a map $u: Z \times I \rightarrow I$ such that $u^{-1}(1)=Z_{0} \times I$ and such that

$$
\widehat{G}^{+}: Z \times I \times I \rightarrow Y \times(-\infty,+\infty]
$$

defined by

$$
\begin{aligned}
\widehat{G}(z, s, t) & =\bar{G}^{+}(z, s,(1-t) \cdot u(z, s)+t) \\
& =\left\{\begin{array}{l}
p G(z, s,(1-t) \cdot u(z, s)+t) \quad \text { if }(z, s, t) \in Z_{1} \times I \times[0,1), \\
F(z, s), \quad \text { if }(z, s, t) \in(Z \times I \times\{1\}) \cup\left(Z_{0} \times I \times I\right),
\end{array}\right.
\end{aligned}
$$

is continuous. It follows from the Continuity Criteria [11, Lemma 3.4] that

$$
\widetilde{F}: Z \times I \times[0,1) \rightarrow X \cup_{p} Y
$$

defined by

$$
\widetilde{F}(z, s, t)= \begin{cases}G(z, s,(1-t) \cdot u(z, s)+t) & \text { if }(z, s, t) \in Z_{1} \times I \times[0,1) \\ \pi_{Y} F(z, s) & \text { if }(z, s, t) \in Z_{0} \times I \times[0,1)\end{cases}
$$

is continuous. One easily checks that $\widetilde{F}$ is a stratified controlled solution of the original problem.

In particular, if $X$ and $Y$ are manifolds and $p: X \rightarrow Y \times \mathbb{R}$ is a manifold approximate fibration, then $c: X \cup_{p} Y \rightarrow Y \times(-\infty,+\infty]$ is a stratified approximate fibration.

\section{References}

[1] A. Beshears, G-isovariant structure sets and stratified structure sets, Ph.D. thesis, Vanderbilt Univ., 1997.

[2] T. A. Chapman, Approximation results in topological manifolds, Mem. Amer. Math. Soc. 251 (1981). 
[3] D. Coram and P. Duvall, Approximate fibrations, Rocky Mountain J. Math. 7 (1977), 275-288.

[4] R. D. Edwards, TOP regular neighborhoods, handwritten manuscript, 1973.

[5] B. Hughes, Approximate fibrations on topological manifolds, Michigan Math. J. 32 (1985), 167-183.

[6] —, Geometric topology of stratified spaces, Electron. Res. Announc. Amer. Math. Soc. 2 (1996), 73-81.

[7] - Stratified path spaces and fibrations, Proc. Roy. Soc. Edinburgh Sect. A 129 (1999), 351-384.

[8] -, Stratifications of mapping cylinders, Topology Appl. 94 (1999), 127-145.

[9] - , The geometric topology of stratified spaces, in preparation.

[10] B. Hughes and A. Ranicki, Ends of Complexes, Cambridge Tracts in Math. 123, Cambridge Univ. Press, Cambridge, 1996.

[11] B. Hughes, L. Taylor, S. Weinberger and B. Williams, Neighborhoods in stratified spaces with two strata, Topology, to appear.

[12] B. Hughes, L. Taylor and B. Williams, Bundle theories for topological manifolds, Trans. Amer Math. Soc. 319 (1990), 1-65.

[13] - - - - Manifold approximate fibrations are approximately bundles, Forum Math. 3 (1991), 309-325.

[14] B. Hughes and S. Weinberger, Surgery and stratified spaces, preprint, http:// xxx.lanl.gov/abs/math.GT/9807156.

[15] F. Quinn, Homotopically stratified sets, J. Amer. Math. Soc. 1 (1988), 441-499.

[16] C. Rourke and B. Sanderson, An embedding without a normal bundle, Invent. Math. 3 (1967), 293-299.

[17] L. Siebenmann, The obstruction to finding a boundary of an open manifold of dimension greater than five, Ph.D. thesis, Princeton Univ., 1965.

Department of Mathematics

Vanderbilt University

Nashville, TN 37240, U.S.A.

E-mail: hughes@math.vanderbilt.edu 